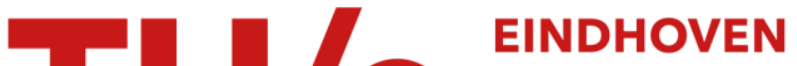 \\ UNIVERSITY OF \\ TECHNOLOGY
}

\section{Isothermal moisture transport in partially saturated porous media}

Citation for published version (APA):

Zanden, van der, A. J. J., Coumans, W. J., Kerkhof, P. J. A. M., \& Schoenmakers, A. M. E. (1996). Isothermal moisture transport in partially saturated porous media. Drying Technology, 14(7\&8), 1525-1542.

https://doi.org/10.1080/07373939608917162

DOI:

10.1080/07373939608917162

Document status and date:

Published: 01/01/1996

\section{Document Version:}

Publisher's PDF, also known as Version of Record (includes final page, issue and volume numbers)

\section{Please check the document version of this publication:}

- A submitted manuscript is the version of the article upon submission and before peer-review. There can be important differences between the submitted version and the official published version of record. People interested in the research are advised to contact the author for the final version of the publication, or visit the $\mathrm{DOI}$ to the publisher's website.

- The final author version and the galley proof are versions of the publication after peer review.

- The final published version features the final layout of the paper including the volume, issue and page numbers.

Link to publication

\section{General rights}

Copyright and moral rights for the publications made accessible in the public portal are retained by the authors and/or other copyright owners and it is a condition of accessing publications that users recognise and abide by the legal requirements associated with these rights.

- Users may download and print one copy of any publication from the public portal for the purpose of private study or research.

- You may not further distribute the material or use it for any profit-making activity or commercial gain

- You may freely distribute the URL identifying the publication in the public portal.

If the publication is distributed under the terms of Article 25fa of the Dutch Copyright Act, indicated by the "Taverne" license above, please follow below link for the End User Agreement:

www.tue.nl/taverne

Take down policy

If you believe that this document breaches copyright please contact us at:

openaccess@tue.nl

providing details and we will investigate your claim. 


\title{
ISOTHERMAL MOISTURE TRANSPORT IN PARTIALLY SATURATED POROUS MEDIA
}

\author{
A.J.J. van der Zanden, W.J. Coumans, P.J.A.M. Kerkhof \\ and A.M.E. Schoenmakers \\ Laboratory of Separation Technology \\ Eindhoven University of Technology, P.O. Box 513 \\ - $5600 \mathrm{MB}$, Eindhoven, The Netherlands
}

Keywords: clay; diffusion; diffusion coefficient; drying; permeability.

\begin{abstract}
The prediction of a hydrodynamic model for the isothermal transport of liquid in partially saturated porous media is compared with experimentally obtained values of water transport in clay. The transport obeys the diffusion equation. The diffusion coefficient is described as a function of porosity, permeability and pore size distribution. The comparison indicates that the model needs some refinement.
\end{abstract}

\section{Introduction}

In many practical problems the process of fluid flow through porous media is involved. In the case of a completely saturated porous medium the 
flow is well described with Darcy's law. In the case the porous medium is only partially saturated with liquid the flow through this porous medium is much more complicated. This kind of flow has been described by Krischer \& Kast (1978) in the case that the porous material can be regarded as a bundle of capillaries. Their result is that the diffusion coefficient can be predicted as a function of the moisture content.

The aim of this article is to put forward a hydrodynamic model which also describes the flow of liquid through partially saturated porous media and which is based on a fundamental, hydrodynamic approach (section $2 \& 3$ ). It does not assume the porous material to be a bundle of capillaries but introduces a parameter to incorporate all effects due to the deviations from the ideal poiseuille flow through capillaries. The comparison with measurements available in the literature suggests that the model needs further study (section 4).

\section{Description of a Porous Material}

Most porous materials have a very intricate nature because of the whimsicalness of the solid structure and the chemical inhomogeneity of the solid when seen on small length scales (of the order of the pores). To arrive at a tractable theory describing moisture transport, the porous material is described with a few easy to handle parameters. One of the parameters used below is the permeability $k_{\infty}$ of the saturated porous 
material which predicts the flow through the porous material when a pressure gradient $d P_{l} / d x$ is applied:

$$
q=-\frac{k_{\infty}}{\mu} \frac{d P_{l}}{d x},
$$

where $\mu$ is the dynamic viscosity of the liquid which flows through the pores and $q$ the superficial liquid velocity [Greenkorn (1983)]. Equation (1) is known as Darcy's law.

Another parameter used in the description of the porous material is the porosity $\epsilon$ which is the volume fraction of the material which is occupied by the pores.

The last parameter is the pore size distribution. It is convenient here to describe the pore size distribution as a cumulative volume fraction $C(R)$ as a function of the pore radius $R . C(R)$ is the fraction of the volume of the pores which have a radius smaller than $R$. It is clear that there exist two limits:

$$
\lim _{R \rightarrow 0} C(R)=0
$$

and

$$
\lim _{R \rightarrow \infty} C(R)=1
$$

The fraction $f(R) d R$ of the volume of the pores which have a radius between $R$ and $R+d R$ (with $d R$ small) is 


$$
f(R) d R=\frac{d C(R)}{d R} d R
$$

$f$ is called the pore size distribution function.

\section{Moisture Transport in Partially Saturated Porous Media}

\section{Static Situation}

A plug of liquid inside a capillary of variable cross section will occupy that part of the capillary which has the smallest cross section due to the capillary forces. For instance in the non static situation as depicted in figure 1 the pressure $P_{2}$ is larger than $P_{1}$ (Laplace' law) which will make the plug move to the right until $P_{1}$ equals $P_{2}$ and the condition mentioned above is met. When the moisture content of a porous material decreases, the first pores that become "dry" are the largest pores. This has also been observed by Engøy, Feder \& Jøssang (1991) in a drying experiment. If a porous material is partially filled with liquid only the pores with a radius below $R_{f}$ are filled where

$$
\epsilon C\left(R_{f}\right)=\frac{V_{l}}{V}
$$

with $V_{l}$ and $V$ the volume of respectively the liquid and the material. The pressure $P_{l}$ in the liquid is then

$$
P_{l}=-\frac{2 \sigma}{R_{f}}
$$




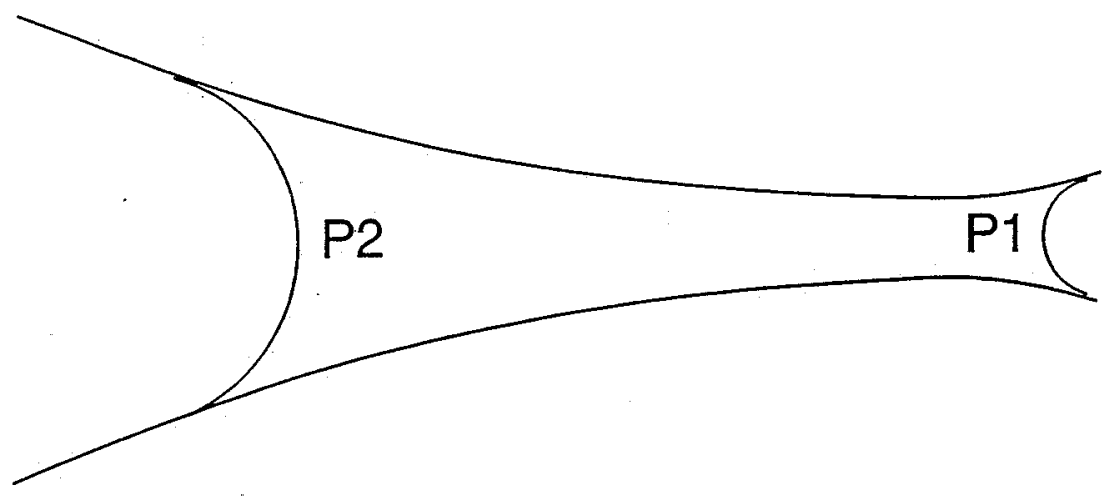

Figure 1. Liquid inside a porous material will flow towards the places where it will occupy the smallest pores in the porous material.

where $\sigma$ is the surface tension (the pressure in the vapour and contact angle are both put zero for convenience).

\section{Dynamic Situation}

When differences in moisture content exist in a porous material, there are differences in $R_{f}$ and according to equation 6 different pressures in the liquid. The result will be transport of liquid through this porous material according to equation 1 where $k_{\infty}$ is now substituted by $k$ the effective permeability of the partially saturated porous material. This effective permeability will be modelled below.

The flux $\varphi$ through one pore in the porous material is (in analogy with a poiseuille type of flow, Batchelor (1967)) modelled as 


$$
\varphi=-F(R) \frac{R^{4} \pi}{8 \mu} \frac{d P_{l}}{d x}
$$

where $F(R)$ accounts for the deviation of the ideal poiseuille flow, due for instance to the tortuosity or the shape of the pores. Therefore equation 7 describes not only the flow in capillaries but also in pores of whatever shape. The number of the pores with a radius between $R$ and $R+d R$ (per unit surface) is

$$
\frac{\epsilon f(R) d R}{\pi R^{2}}
$$

The flux $d q$ through porous material due to the channels with a pore radius between $R$ and $R+d R$ follows from (7) and (8):

$$
d q=-\epsilon f(R) d R F(R) \frac{R^{2}}{8 \mu} \frac{d P_{l}}{d x} .
$$

From (1) and (9) follows

$$
k_{\infty}=\int_{0}^{\infty} \epsilon f(R) F(R) \frac{R^{2}}{8} d R .
$$

On the assumption that $F(R)$ is equal for all $R$ equation 10 becomes

$$
k_{\infty}=\frac{\epsilon F}{8} \int_{0}^{\infty} f(R) R^{2} d R .
$$

$F$ can be determined from equation 11 once $k_{\infty}, \epsilon$ and $f(R)$ [or $\left.C(R)\right]$ are measured. 
In a partially saturated porous medium the channels filled with vapour are supposed not to make a contribution to the transport of moisture through the porous material. The relative humidity in the "empty" pores is $100 \%$ and then the vapour in the pores will not flow through the pores because of the absence of any pressure or concentration differences. At a drying boundary the relative humidity can become smaller but let it be explicitly stated that it is not the aim of this article to describe a boundary condition. Only the filled pores then contribute to the flow of moisture in the porous medium. The permeability of a partially saturated porous medium is then only formed by the "filled" pores. For the effective permeability $k$ as a function of $R_{f}$ is then written in analogy with equation 11

$$
k\left(R_{f}\right)=\frac{\epsilon F}{8} \int_{0}^{R_{f}} f(R) R^{2} d R .
$$

The fraction $k\left(R_{f}\right) / k_{\infty}$ is known as the relative permeability [Bear \& Bachmat (1991)].

Below a relation will be derived for the diffusion coefficient which describes the liquid transport inside the porous material. The driving agent for the liquid transport is the gradient in the pressure in the liquid which is caused by a gradient in the moisture content-Differentiating equation (6) gives

$$
\frac{d P_{l}}{d x}=\frac{2 \sigma}{R_{f}^{2}} \frac{d R_{f}}{d x}
$$


Differentiating equation (5) gives

$$
\epsilon f\left(R_{f}\right) \frac{d R_{f}}{d x}=\frac{1}{V} \frac{d V_{l}}{d x} .
$$

Substituting (14) in (13) gives

$$
\frac{d P_{l}}{d x}=\frac{2 \sigma}{R_{f}^{2}} \frac{1}{\epsilon f\left(R_{f}\right)} \frac{d\left(V_{l} / V\right)}{d x} .
$$

The superficial fluid velocity through a partially saturated porous medium is

$$
q=\frac{-k\left(R_{f}\right)}{\mu} \frac{d P_{l}}{d x}=\frac{-\epsilon F_{\sigma}}{4 \mu R_{f}^{2} \in f\left(R_{f}\right)} \frac{d\left(V_{l} / V\right)}{d x} \int_{0}^{R_{f}} f(R) R^{2} d R
$$

A diffusion coefficient $D$ can be defined with

$$
q=-D \frac{d\left(V_{l} / V\right)}{d x}
$$

From equations (11), (16) and (17) follows

$$
D=\frac{2 k_{\infty} \sigma}{\mu \in R_{f}^{2} f\left(R_{f}\right)} \frac{\int_{0}^{R_{f}} f(R) R^{2} d R}{\int_{0}^{\infty} f(R) R^{2} d R} .
$$

From the considerations as outlined in the subsection "static situation" it is clear that what must be continuous over a transition from one porous medium to another is the pressure $P_{l}$. According to equation 6 this 
means that $R_{f}$ must be continuous. Because different porous media have different $C(R)$ it is very unlikely that at an interface between two porous media the moisture content, $V_{l} / V$, is continuous.

When gravity plays a role an extra pressure gradient is present due to the hydrostatic pressure difference. The flux $\bar{q}$ (a vector) through a porous medium is then described with

$$
\bar{q}=\frac{-k\left(R_{f}\right)}{\mu} \nabla\left(P_{l}+\rho g x\right)
$$

where $\rho$ is the density of the liquid and $g$ the gravitational acceleration which here points in the negative $x$-direction.

\section{Comparison with Experiments}

Using a scanning neutron radiography technique diffusion coefficients have been measured by Pel, Ketelaars, Adan \& van Well (1993). Ketelaars (1992) measured the diffusion coefficient of water (using the same technique) in three types of clay. The pore size distribution was also measured while the permeability was unfortunately not reported. Here one of the reported clays will be used to test the theory outlined above. Clay C (in the terminology of Ketelaars) was chosen because of the wide distribution of its pore diameter and because $C(R)$ can be approximated analytically rather easily and accurately. In figure 2 the function $C$ is given as a function of the pore radius $(R)$ as it was measured by Ketelaars using 


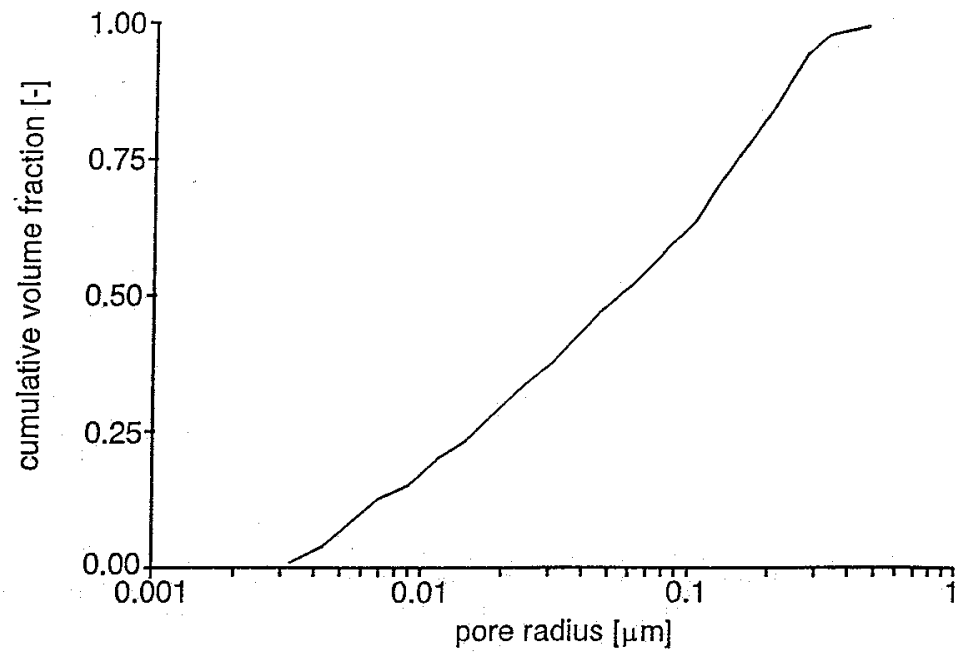

Figure 2. $\quad C(R)$ as a function of the pore radius $R$ as was measured by Ketelaars.

mercury porosimetry on dried clay samples. For $R$ between $4 \cdot 10^{-9}$ and $3.5 \cdot 10^{-7} \mathrm{~m} C(R)$ can be approximated with

$$
C(R)=\frac{\ln (R)-\ln \left(4 \cdot 10^{-9}\right)}{\ln \left(3.5 \cdot 10^{-7}\right)-\ln \left(4 \cdot 10^{-9}\right)}
$$

(a straight line in figure 2), from which follows

$$
f(R)=\frac{1}{4.47 R}
$$

From (5) and (20) follows

$$
R_{f}=\exp \left(\frac{4.47 V_{l}}{\epsilon V}+\ln \left(4 \cdot 10^{-9}\right)\right)
$$


With equation (21) equation (18) can be written as

$$
D=\frac{8.94 k_{\infty} \sigma}{\mu \in R_{f}} \frac{\int_{4 \cdot 10^{-9}}^{R_{f}} R d R}{\int_{4 \cdot 10^{-9}}^{3.5 \cdot 10^{-7}} R d R}
$$

or

$$
D=\frac{8.94 k_{\infty} \sigma}{\mu \in R_{f}} \frac{R_{f}^{2}-\left(4 \cdot 10^{-9}\right)^{2}}{\left(3.5 \cdot 10^{-7}\right)^{2}-\left(4 \cdot 10^{-9}\right)^{2}} .
$$

The permeability of saturated clay was measured with a set up as depicted in figure 3 . Out of a volume $\left(\approx 610^{-3} \mathrm{~m}^{3}\right)$ an amount of air could be pumped such as to create a lower pressure under the clay sample than outside. The pressure difference over the clay sample (typically $10^{4} \mathrm{~N} \mathrm{~m}^{-2}$ ) causes air to flow through the sample. After the valve is closed the pressure in the volume will slowly increase. From the pressure as a function of time the mass flux of air through the sample was calculated. The permeability follows from

$$
\frac{P_{o}^{2}-P_{i}^{2}}{L}=\frac{2 R_{c} T \mu_{a i r} G}{k_{\infty} M}
$$

with $P_{o}$ and $P_{i}$ the pressure outside respectively inside the set up, $L$ the thickness of the sample $[\approx 3 \mathrm{~cm}], R_{c}$ the gas constant, $T$ temperature, $\mu_{\mathrm{air}}$ viscosity of the air, $G$ the mass flux of air and $M$ the molar weight of air 


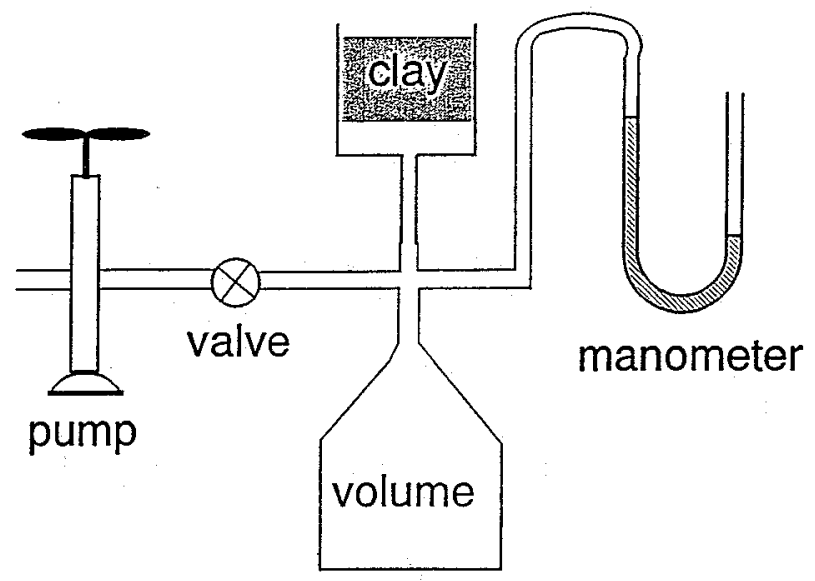

Figure 3. Experimental set up used to measure the permeability of a clay sample.

(Perry 1973). The measurements were performed with three disk shaped samples of clay $\mathrm{C}$ having a diameter of approximately $5 \mathrm{~cm}$. The permeability was $2.910^{-16}, 8.610^{-17}$ and $2.210^{-16} \mathrm{~m}^{2}$ which shows a large scattering probably due to sample preparation.

In figure 4 the diffusion coefficient as predicted by the model (line 1) is compared with the experiments of Ketelaars (squares). In the model the porosity was taken to be 0.3 and the permeability as the average of the measurements $\left(1.9910^{-16} \mathrm{~m}^{2}\right)$. The surface tension and viscosity were taken respectively $73: 10^{-3} \mathrm{~N} \mathrm{~m}^{-1}$ and $10^{-3} \mathrm{~N} \mathrm{~s} \mathrm{~m}^{-2}$. The deviation of the model from the experiment is extremely large. If $k_{\infty}$ has a value of 1.41$2 \cdot 10^{-18} \mathrm{~m}^{2}$ a good agreement between the model and the experiments results 


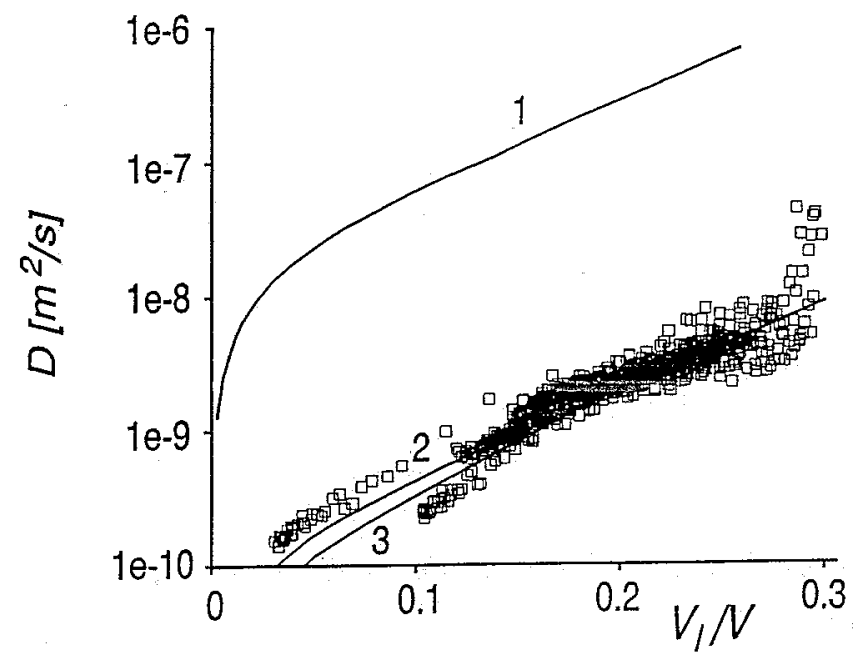

Figure 4. The diffusion coefficient as predicted by the model as a function of the moisture content (line 1) compared with the experiments of Ketelaars (squares). Line 2 and 3 are the prediction of the model when the permeability is fit to obtain a good agreement with the experiments (porosity respectively 0.3 and 0.25 ).

(porosity also 0.3 ). This is depicted as line 2. Changing the value of $k_{\infty}$ makes the line move in a vertical direction [see equation (24)].

Ketelaars reported for clay $C$ the maximum moisture content of 0.14 $\mathrm{kg}$ water per $\mathrm{kg}$ dry clay and the specific volume of dry clay as $5.610^{4} \mathrm{~m}^{3}$ per $\mathrm{kg}$ dry clay. This would imply a porosity of 0.25 . This is contrary to the reported measurements of the diffusion coefficients which are reported for even as high a liquid volume fraction as 0.29 . During the drying some 
shrinkage must have occurred. However, for the porosity 0.25 the predictions of the model presented here are also depicted in figure 3 (line 3). For $k_{\infty}$ the value of $6.55 \cdot 10^{-19} \mathrm{~m}^{2}$ was needed to obtain a good fit.

\section{Conclusions and Discussion}

A model has been presented which describes the fluid flow through partially saturated porous media. This model needs only input parameters which can be measured rather easily and independently.

What immediately catches the eye is the striking deviation of the experimental results from the predictions of the model. There are two candidates that can introduce these deviations, i.e. the capillary pressure and the effective permeability. As to the first it seems very unlikely to introduce large deviations from the model because the geometrical shape of the mercury interface during imbibition is identical to the water interface during drainage if it is assumed that the contact angles are $180^{\circ}$ and $0^{\circ}$ respectively. Small deviations in contact angle will introduce only small deviations in the capillary pressure. The large deviation between prediction and measurement must therefore be sought in the way the permeability is modelled. Here two possible explanations are put forward. First, the notion that there exists a sharp distinction between empty and filled pores is probably a little naive. The pores are not round and the liquid may flow through the "corners" of "empty" pores. Second it is assumed that the correction factor $F$ is independent of pore radius. This needs not to be true. 
For instance the poiseuille flow through capillaries is a fully developed, parabolic flow pattern. In the pores the "plugs" of liquid are finite. The factor $F$ must incorporate these effects. It is imaginable that these effects are radius dependent. If it is assumed that $F$ is radius independent then $F$ can be calculated with equation 11 as 0.39 . For a one phase flow this gives no reason for suspicion.

For this model to be valid it is necessary that the liquid can seep through the pores. It is conceivable that there exist porous media in which very small drops of liquid are present but where the liquid cannot flow because there is no percolating path through the liquid from one side of the porous material to the other side. On the other hand it is also imaginable that there is no percolating path in the vapour phase in an almost totally saturated porous medium. The vapour is then present in the form of bubbles. The assumption of a uniform pressure in the vapour phase (put zero in this article) needs not to be valid any longer in such a case.

The model presented here can easily be extended towards the case of another contact angle or to the case where the viscosity of the vapour (or a second liquid) cannot be neglected.

probably a little naive. The pores are not round and the liquid may flow through the "corners" of "empty" pores. Second it is assumed that the correction factor $F$ is independent of pore radius. This needs not to be true. For instance the poiseuille flow through capillaries is a fully developed, parabolic flow pattern. In the pores the "plugs" of liquid are finite. The factor $F$ must incorporate these effects. It is imaginable that these effects 
are radius dependent. If it is assumed that $F$ is radius independent then $F$ can be calculated with equation 11 as 0.39 . For a one phase flow this gives no reason for suspicion.

For this model to be valid it is necessary that the liquid can seep through the pores. It is conceivable that there exist porous media in which very small drops of liquid are present but where the liquid cannot flow because there is no percolating path through the liquid from one side of the porous material to the other side. On the other hand it is also imaginable that there is no percolating path in the vapour phase in an almost totally saturated porous medium. The vapour is then present in the form of bubbles. The assumption of a uniform pressure in the vapour phase (put zero in this article) needs not to be valid any longer in such a case.

The model presented here can easily be extended towards the case of another contact angle or to the case where the viscosity of the vapour (or a second liquid) cannot be neglected.

For a further discussion see also van der Zanden (1995).

\section{NOTATION}

C cumulative volume fraction of the pores

$D$ diffusion coefficient $\left[\mathrm{m}^{2} \mathrm{~s}^{-1}\right]$

$f$ pore size distribution function $\left[\mathrm{m}^{-1}\right]$

$F \quad$ correction factor

$g$ gravitational acceleration [m s $\mathrm{m}^{-2}$ ]

$G$ air mass flux $\left[\mathrm{kg} \mathrm{m}^{-2} \mathrm{~s}^{-1}\right]$

$k \quad$ permeability $\left[\mathrm{m}^{2}\right]$ 
$L \quad$ thickness of the clay sample [m]

$M \quad$ molar weight of air $\left[\mathrm{kg} \mathrm{kmol}^{-1}\right]$

$P \quad$ pressure $\left[\mathrm{N} \mathrm{m}^{-2}\right]$

$q \quad$ average fluid velocity $\left[\mathrm{m} \mathrm{s}^{-1}\right.$ ]

$R$ pore radius [m]

$R_{c} \quad$ gas constant $\left[\mathrm{kg} \mathrm{m}^{2} \mathrm{~s}^{-2} \mathrm{kmol}^{-1} \mathrm{~K}^{-1}\right]$

$T$ temperature [K]

$V_{l} \quad$ volume of the liquid $\left[\mathrm{m}^{3}\right]$

$V \quad$ volume of the drying material $\left[\mathrm{m}^{3}\right]$

$x \quad$ place coordinate [m]

Greek symbols

$\epsilon$ porosity

$\mu \quad$ viscosity $\left[\mathrm{N} \mathrm{m}^{-2} \mathrm{~s}\right]$

$\rho$ density $\left[\mathrm{kg} \mathrm{m}^{-3}\right]$

$\sigma \quad$ surface tension $\left[\mathrm{N} \mathrm{m}^{-1}\right]$

$\varphi \quad$ flux through one pore $\left[\mathrm{m}^{3} \mathrm{~s}^{-1}\right]$

Subscripts

air vapour phase

$f \quad$ largest filled pores

$l \quad$ liquid

$\infty \quad$ saturated material

\section{References}

Batchelor, G.K., 1967, An introduction to fluid dynamics, Cambridge University Press, pp 180.

Bear, J. and Bachmat, Y., 1991, Introduction to modeling of transport phenomena in porous media, pp. 360 , Kluwer academic publishers.

Engøy, T., Feder, J. and Jøssang, T., 1991, Counter-flow in drying of competing pores, Physica Scripta T38, pp. 99-102. 
Greenkorn, R.A., 1983, Flow phenomena in porous media: fundamentals and applications in petroleum, water, and food production, pp. 5, Marcel Dekker, INC.

Ketelaars, A.A.J., 1992, Drying deformable media: kinetics, shrinkage and stresses, Ph.D. Thesis, University of Technology Eindhoven, Eindhoven, The Netherlands, pp. 107.

Krischer, O. and Kast, W., 1978, Die wissenschaftlichen Grundlagen der Trocknungstechnik, Dritte Auflage, chapter 5.9.4, pp. 215-222, SpringerVerlag Berlin Heidelberg New York.

Pel, L., Ketelaars, A.A.J., Adan, O.C.G. and van Well, A.A., 1993, Determination of moisture diffusivity in porous media using scanning neutron radiography, Int. J. Heat Mass Transfer 36(5), pp. 1261-1267.

Perry, R.H. and Chilton, C.H., 1973, Chemical Engineers' Handbook, fifth edition, McGraw-Hill, pp. 5-54.

van der Zanden, A.J.J., 1995, A possible revision of the results of a model for moisture transport in partially saturated porous media, Drying Technology 13(8-9), pp. 2227-2231. 